\title{
Clinical Proteomic Tumor Analysis Consortium
}

National Cancer Institute

\section{Source}

National Cancer Institute. Clinical Proteomic Tumor Analysis Consortium. NCI Thesaurus.

Code C157175.

A national effort to accelerate the understanding of the molecular basis of cancer through the application of large-scale proteome and genome analysis, or proteogenomics. 